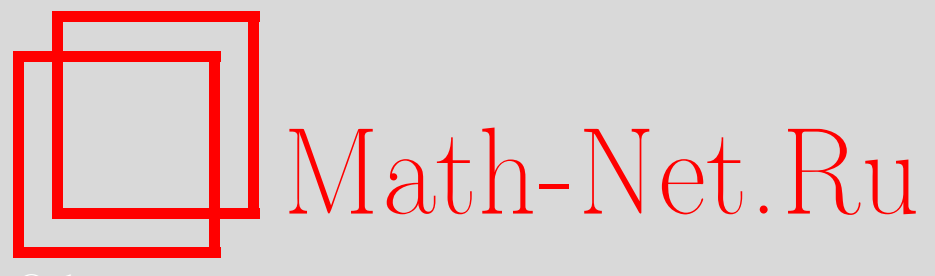

Т. Д. Блайвас, Асимптотика сложности интервального поиска на булевом кубе в классе сбалансированных деревьев, Дискрет. матем., 2004, том 16, выпуск 4, 65-78

DOI: https://doi.org/10.4213/dm176

Использование Общероссийского математического портала Math-Net.Ru подразумевает, что вы прочитали и согласны с пользовательским соглашением http: //www.mathnet.ru/rus/agreement

Параметры загрузки:

IP : 35.173 .219 .149

26 апреля 2023 г., 05:04:17

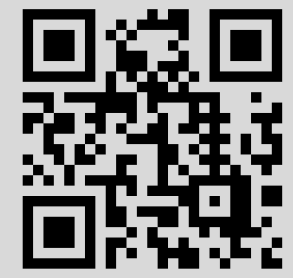




\title{
Асимптотика сложности интервального поиска на булевом кубе в классе сбалансированных деревьев
}

\author{
() 2004 г. Т. Д. Блайвас
}

\begin{abstract}
Для задачи интервального поиска на булевом кубе исследуется асимптотическое поведение среднего времени поиска в классе сбалансированных древовидных схем на последовательностях натуральных чисел $\left\{k_{i}\right\}$ в предположении, что $k_{i}-$ мощность баз данных, $i=1,2, \ldots$ Показано, что для разных последовательностей асимптотическое поведение может быть разным. Полностью описан класс возможных асимптотических поведений.

Работа выполнена при поддержке Российского фонда фундаментальных исследований, проект 01-01-00748.
\end{abstract}

\section{1. Введение}

В работе рассматривается следующая задача информационного поиска. Пусть $V$ - некоторое подмножество $n$-мерного булева куба $B_{2}^{n}$, называемое библиотекой. На булевом кубе берется произвольный интервал $(u, w)$, где $u=\left(u_{1}, \ldots, u_{n}\right), w=\left(w_{1}, \ldots, w_{n}\right)$ и $u \preccurlyeq w$, то есть $u_{i} \leqslant w_{i}, i=1, \ldots, n$. Требуется определить все такие элементы $y \in V$, $y=\left(y_{1}, \ldots, y_{n}\right)$, называемые записями, для которых $u \preccurlyeq y \preccurlyeq w$.

Приведем интерпретацию данной задачи. Допустим, что имеется частично разгаданный кроссворд, в котором все слова имеют одинаковую длину. Отгадываем слово, в котором известны не все буквы. Требуется найти в словаре такие слова, которые потенциально могут быть разгадываемым словом.

Задачу можно решить, если на каждом шаге алгоритма проверять условие $u_{j} \leqslant y_{j} \leqslant w_{j}, j \in\{1, \ldots, n\}$, для фиксированного набора компонент записи, причем номера компонент одинаковы для всех записей на одном шаге алгоритма.

Классу таких алгоритмов удобно сопоставить сбалансированные информационные деревья. В данной работе описана процедура построения оптимальных сбалансированных информационных деревьев, решающих задачу интервального поиска на булевом кубе. Получены оценки сложности этих деревьев. Исследовано асимптотическое поведение сложности на растущих последовательностях натуральных чисел $\left\{k_{i}\right\}$ в предположении, что $k_{i}$ - мощность баз данных, $i=1,2, \ldots$ Показано, что для разных последовательностей асимптотическое поведение может быть разным. Полностью описан класс возможных асимптотических поведений. Результаты данной работы анонсированы в [1]. 


\section{2. Основные понятия и формулировка результатов}

Мы будем использовать терминологию и обозначения работы [2], но поскольку в данной работе рассматриваются только древовидные схемы, здесь будет приведена несколько упрощенная версия понятия информационного графа.

Если $X$ - множество символов запросов с заданным на нем вероятностным пространством $\langle X, \sigma, \mathbf{P}\rangle$, где $\sigma$ - алгебра подмножеств множества $X, \mathbf{P}$ - вероятностная мера на $\sigma ; Y$ - множество символов данных (записей), $\rho-$ бинарное отношение на $X \times Y$, называемое отношением поиска, то пятерка $S=\langle X, Y, \rho, \sigma, \mathbf{P}\rangle$ называется типом. Тройка $I=\langle X, V, \rho\rangle$, где $V$ - некоторое конечное подмножество множества $Y$, называемое библиотекой, называется задачей информационного поиска (ЗИП) типа $S$. Содержательно ЗИП $I=\langle X, V, \rho\rangle$ состоит в перечислении для произвольно взятого запроса $x \in X$ всех тех и только тех записей $y \in V$, для которых $x \rho y$. Если $\mathscr{F}$ - множество символов одноместных предикатов, определенных на $X$, то F्F называется базовым множеством, оно описывает множество элементарных операций, используемых при решении задачи информационного поиска.

Над базовым множеством F्ғ определяется информационный граф (ИГ). В конечной многополюсной ориентированной сети выбирается вершина-полюс, называемая корнем. Остальные полюсы называются листьями и им приписываются записи из $Y$. Ребрам ИГ приписываются предикаты из множества $\mathscr{F}$. Таким образом нагруженную многополюсную ориентированную сеть называют информационным графом над базовым множеством F्F. Затем определяется функционирование ИГ. Предикатное ребро проводит запрос $x \in X$, если предикат ребра истинен на $x$; ориентированная цепочка ребер проводит $x$, если каждое ребро цепочки проводит $x$; запрос $x$ проходит в вершину $\beta$ ИГ, если существует ориентированная цепь, ведущая из корня в вершину $\beta$, которая проводит $x$; запись $y$, приписанная листу $\alpha$, попадает в ответ ИГ на $x$, если $x$ проходит в лист $\alpha$. Ответом ИГ $U$ на запрос $x$ называют множество записей, попавших в ответ $U$ на $x$, и обозначают его $\mathscr{F}_{U}(x)$. Эту функцию $\mathscr{F}_{U}(x)$ считают результатом функционирования ИГ $U$.

ИГ $U$ разрешает ЗИП $I=\langle X, V, \rho\rangle$, если

$$
\mathscr{L}_{U}(x)=\{y \in V: x \rho y\}
$$

Вводится сложность ИГ. Предикат $\varphi_{\beta}(x)$ истинный на $x$, если $x$ проходит в вершину $\beta$, и ложный в противном случае, называется функцией фильтра вершины $\beta$. Сложностью ИГ $U$ на запросе $x \in X$ называется число

$$
T(U, x)=\sum_{\beta \in \mathscr{R}} \psi_{\beta} \varphi_{\beta}(x),
$$

где $\mathscr{R}$ - множество вершин ИГ $U, \psi_{\beta}$ - количество ребер, исходящих из вершины $\beta$. Эта величина равна числу функций, вычисленных алгоритмом поиска, определяемым ИГ $U$, на запросе $x$.

Если каждая функция из $\mathscr{F}$ измерима (относительно алгебры $\sigma$ ), то для любого ИГ $U$ над $\mathscr{F}$ функция $T(U, x)$ измерима.

Сложностью ИГ $U$ называется математическое ожидание величины $T(U, x)$, равное

$$
T(U)=\mathbf{M}_{x} T(U, x) .
$$

Она характеризует среднее время поиска. 
Ясно, что

$$
T(U)=\sum_{\beta \in U} \psi_{\beta} \mathbf{P}\left(N_{\varphi_{\beta}}(x)\right) .
$$

Если $f$ - предикат на множестве $X$, то

$$
N_{f}(x)=\{x \in X: f(x)=1\} .
$$

Сложностью ребра, исходящего из вершины $\beta$, назовем число $\mathbf{P}\left(N_{\varphi_{\beta}}(x)\right)$. Согласно (1), сложность ИГ равна сумме сложностей ребер.

Рассмотрим следующую ЗИП. Имеется некоторое $k$-элементное подмножество $n$-мерного булева куба $V \in B_{2}^{n}$ (библиотека). На булевом кубе задан некоторый интервал $(u, w)$, где $u=\left(u_{1}, \ldots, u_{n}\right), w=\left(w_{1}, \ldots, w_{n}\right)$, и $u \preccurlyeq w$, то есть $u_{i} \leqslant w_{i}$, для всех $i=1, \ldots, n$. Требуется определить все элементы $y \in V$, удовлетворяющие условию $u \preccurlyeq y \preccurlyeq w$.

Очевидно, что если $u_{i}=1$ для некоторого $i$, то и $w_{i}=1$, а следовательно, и $y_{i}=1$. Аналогично, если $w_{i}=0$, то и $y_{i}=0$. Таким образом, вышеописанная ЗИП сводится к следующей: есть библиотека $V \in B_{2}^{n},|V|=k$, берем запрос $x=\left(x_{1}, \ldots, x_{n}\right)$, трехзначный вектор, компоненты которого могут быть равны либо 1 , либо 0 , либо 2: если $u_{i}=1$, то $x_{i}=1$, если $w_{i}=0$, то $x_{i}=0$, в противном случае $x_{i}=2$. Для данного запроса $x=\left(x_{1}, \ldots, x_{n}\right)$ требуется найти все $y=\left(y_{1}, \ldots, y_{n}\right) \in V$, для которых $y_{i}=x_{i}$, если $x_{i}=1$ или $x_{i}=0$, и $y_{i}-$ любое из $\{0,1\}$, если $x_{i}=2$.

Получаем тип задач $S_{n}=\left\langle B_{3}^{n}, B_{2}^{n}, \rho, \sigma, P\right\rangle$, где $B_{3}^{n}$ и $B_{2}^{n}-$ трехзначный и двузначный (булевы) кубы, соответственно, $\rho: x \rho y \Longleftrightarrow\left(x_{i}=y_{i}\right) \vee\left(x_{i}=2\right), \sigma-$ множество подмножеств $B_{3}^{n}$, и $\mathbf{P}-$ равномерная вероятностная мера на $B_{3}^{n}$, то есть $\mathbf{P}(x)=3^{-n}$ для любого $x \in B_{3}^{n}$.

Вершину со степенью полуисхода, равной нулю, назовем висячей вершиной. Информационным деревом (ИД) назовем ИГ без циклов, множество листьев которого совпадает с множеством висячих вершин и все ребра которого ориентированы от корня к листьям. Высотой ИД назовем длину максимального пути из корня в лист.

Пусть $\mathbf{X}-$ множество переменных $\left\{x_{1}, \ldots, x_{n}\right\}$. Упорядоченное множество $\mathbf{R}=\left\{\mathbf{X}_{1}, \ldots, \mathbf{X}_{m}\right\}$ будем называть разбиением множества $\mathbf{X}$, если

$$
\bigcup_{i=1}^{m} \mathbf{X}_{i}=\mathbf{X}
$$

и $\mathbf{X}_{i} \cap \mathbf{X}_{j}=\varnothing$ для $i \neq j, i, j=1, \ldots, m$.

Пусть $x \in\{0,1,2\}$ и $y \in\{0,1,2\}$. Определим функцию $x^{y}$, полагая

$$
x^{y}= \begin{cases}x, & \text { если }(y=1) \&(x \neq 2), \\ \bar{x}, & \text { если }(y=0) \&(x \neq 2), \\ 1, & \text { если }(y=2) \vee(x=2),\end{cases}
$$

причем $\bar{x}$ понимается здесь как булево отрицание.

Определим понятие яруса вершин. Вершиной первого яруса будем называть корень. Для любого числа $i$, не большего высоты дерева плюс 1, вершинами $i$-го яруса назовем такие вершины, из которых длина пути в корень равна $i-1$.

Сбалансированным деревом, соответствующим разбиению $\mathbf{R}=\left\{\mathbf{X}_{1}, \ldots, \mathbf{X}_{m}\right\}$ множества $\mathbf{X}$, будем называть любое ИД высоты $m$, удовлетворяющее следующим условиям: 
- из каждой вершины $i$-го яруса вершин, $i=1, \ldots, m-2$, выходит точно $2^{\left|\mathbf{X}_{i}\right|}$ ребер, и если $\mathbf{X}_{i}=\left\{x_{i_{1}}, \ldots, x_{i_{p}}\right\}$, то этим ребрам взаимно однозначно сопоставлены конъюнкщии вида $x_{i_{1}}^{\sigma_{1}} \& \ldots \& x_{i_{p}}^{\sigma_{p}}$, где $\sigma_{i} \in\{0,1\}, i=1, \ldots, p$;

- из каждой вершины $m$-го яруса выходит не более $2^{\left|\mathbf{X}_{m}\right|}$ ребер, и им приписаны конъюнкции (различные для каждой пары ребер, исходящих из одной вершины) вида $x_{m_{1}}^{\sigma_{1}} \& \ldots \& x_{m_{q}}^{\sigma_{q}}$, где $\sigma_{i} \in\{0,1\}, i=1, \ldots, q$, и где $\mathbf{X}_{m}=\left\{x_{m_{1}}, \ldots, x_{m_{q}}\right\}$.

Множество задач $I=\left\langle B_{3}^{n}, V, \rho\right\rangle$ типа $S_{n}$, где $|V|=k$, обозначим через $\mathscr{I}(n, k)$. Множество сбалансированных деревьев высоты $h$ с $k$ висячими вершинами, соответствующих разбиениям множества $\mathbf{X}=\left\{x_{1}, \ldots, x_{n}\right\}$ на $h$ подмножеств, обозначим $\mathscr{D}_{b}(n, k, h)$.

Определим сложность ЗИП $I \subseteq \mathscr{I}(n, k)$ над множеством $\mathscr{D}_{b}(n, k, h)$ равенством

$$
T_{d}(I, h)=\inf _{D \in \mathscr{D}_{b}(n, k, h)} T(D) .
$$

Сложностью задачи $I \subseteq \mathscr{I}(n, k)$ назовем

$$
T_{d}(I)=\min _{h} T(I, h) .
$$

ИД $D$, решающее задачу $I$, назовем оптимальным, если

$$
T_{d}(I)=T(D)
$$

В силу конечности множества сбалансированных деревьев, решающих задачу типа $\left\langle B_{3}^{n}, B_{2}^{n}, \rho\right\rangle$, оптимальное дерево существует.

Введем обозначения

$$
c_{1}=\left(\log _{4 / 3} 2\right)\left(\frac{7}{36} \log _{4 / 3} \frac{3}{2}\right)^{\log _{2}(2 / 3)}, \quad c_{2}=2\left(\frac{7}{36}\right)^{\log _{2}(2 / 3)} .
$$

Теорема 1. Для любой $3 И П I \subseteq \mathscr{I}(n, k)$

$$
c_{1} k^{2-\log _{2} 3}-\frac{36}{7} \leqslant T_{d}(I) \leqslant c_{2} k^{2-\log _{2} 3}-\frac{34}{7} .
$$

Теорема 2. Для любого числа $a \in\left[c_{1}, c_{2}\right]$ и любого действительного $\varepsilon>0$ существует $b \in(a-\varepsilon, a+\varepsilon)$ такое, что существует подпоследовательность $\left\{k_{i}\right\}$ натурального ряда, для которой

(1) $k_{i} \rightarrow \infty$ npu $i \rightarrow \infty$,

(2) для любой последовательности задач информачионного поиска $I_{i} \in \mathscr{T}\left(n, k_{i}\right)$ при $i \rightarrow \infty$

$$
T_{d}\left(I_{i}\right) \sim b k_{i}^{2-\log _{2} 3}
$$

\section{3. Построение оптимальных сбалансированных деревьев}

Рассмотрим задачу интервального поиска $I=\left\langle B_{3}^{n}, V, \rho\right\rangle$, где $|V|=k$. Пусть $D-$ оптимальное сбалансированное дерево, соответствующее разбиению $\mathbf{R}=\left\{\mathbf{X}_{1}, \ldots, \mathbf{X}_{h+1}\right\}$ и решающее задачу $I$. Везде далее

$$
l_{i}=\sum_{j=1}^{i}\left|\mathbf{X}_{i}\right|, \quad i=1, \ldots, h .
$$


Для удобства введем в рассмотрение вспомогательный параметр $l_{0}=0$. Сложность сбалансированного дерева $D$ равна

$$
T(D)=2^{l_{1}}+2^{l_{2}}\left(\frac{2}{3}\right)^{l_{1}}+\ldots+k\left(\frac{2}{3}\right)^{l_{h}} .
$$

Это следует из определения сложности ИГ и структуры сбалансированного дерева, так как если $\beta$ - вершина $i$-го яруса, то $\mathbf{P}\left(\mathcal{N}_{\varphi_{\beta}}(x)\right)=(2 / 3)^{l_{i}}$, а полустепень исхода каждой вершины этого яруса равна $\psi_{\beta}=2^{\left|X_{i+1}\right|}=2^{l_{i+1}-l_{i}}$, всего же вершин на этом ярусе $2^{l_{i}}$. Следовательно, на каждом ярусе с номером $i$, находится $2^{l_{i}}$ ребер, а вероятность попасть в вершины, из которых исходят эти ребра равна $(2 / 3)^{l_{i-1}}, i=1, \ldots, h$.

Лемма 1. Если ИД D, соответствующее разбиению $\mathbf{R}=\left\{\mathbf{X}_{1}, \ldots, \mathbf{X}_{h+1}\right\}$ и решающее задачу $I \in \mathscr{I}(n, k)$, является оптимальньм, то для всех $i=1, \ldots, h$ справедливо неравенство $\left|\mathbf{X}_{i}\right| \leqslant 2$.

Доказательство. Пусть для некоторого $i \in\{0,1, \ldots, h-1\}$ выполнено неравенство $\left|\mathbf{X}_{i+1}\right|=l_{i+1}-l_{i}>2$.

Пусть $\mathbf{X}_{i+1}=\left\{x_{j_{1}}, \ldots, x_{j_{l_{1+1}-l_{i}}}\right\}$. Построим следующее разбиение $\mathbf{R}_{1}$ множества $\mathbf{X}$ : если $\mathbf{R}=\left\{\mathbf{X}_{1}, \ldots, \mathbf{X}_{i}, \mathbf{X}_{i+1}, \ldots, \mathbf{X}_{h_{0}+1}\right\}$, то $\mathbf{R}_{1}=\left\{\mathbf{X}_{1}, \ldots, \mathbf{X}_{i}, \mathbf{X}_{i+1}^{\prime}, \mathbf{X}_{i+2}^{\prime}, \ldots, \mathbf{X}_{h+1}^{\prime}\right\}$, где $\mathbf{X}_{i+1}^{\prime}=\left\{x_{j_{1}}\right\}, \mathbf{X}_{i+2}^{\prime}=\left\{x_{j_{2}}, \ldots, x_{j_{l_{i+1}-l_{i}}}\right\}$ и $\mathbf{X}_{p}^{\prime}=\mathbf{X}_{p-1}$ при $p=i+3, \ldots, h+2$. Рассмотрим сбалансированное дерево $D_{1}$, соответствующее разбиению $\mathbf{R}_{1}$ и решающее задачу $I$. Поскольку дерево $D$ оптимально, должно выполняться неравенство $T(D)-T\left(D_{1}\right) \leqslant 0$. Нетрудно заметить, что сложности $D$ и $D_{1}$ отличаются только слагаемыми, соответствующими ярусу с номером $i+1$ исходного дерева (это слагаемое есть $2^{l_{i+1}}(2 / 3)^{l_{i}}$ ) и ярусам с номерами $i+1$ и $i+2$ нового дерева (эти слагаемые суть $\left.2^{l_{i}+1}(2 / 3)^{l_{i}}+2^{l_{i+1}}(2 / 3)^{l_{i}+1}\right)$. Значит,

$$
\begin{aligned}
T(D)-T\left(D_{1}\right) & =2^{l_{i+1}}\left(\frac{2}{3}\right)^{l_{i}}-2^{l_{i}+1}\left(\frac{2}{3}\right)^{l_{i}}-2^{l_{i+1}}\left(\frac{2}{3}\right)^{l_{i}+1} \\
& =2^{l_{i+1}}\left(\frac{2}{3}\right)^{l_{i}}\left(1-\frac{2}{2^{l_{i+1}-l_{i}}}-\frac{2}{3}\right) \\
& =2^{l_{i+1}}\left(\frac{2}{3}\right)^{l_{i}}\left(\frac{1}{3}-\frac{2}{2^{l_{i+1}-l_{i}}}\right) \\
& >2^{l_{i+1}}\left(\frac{2}{3}\right)^{l_{i}}\left(\frac{1}{3}-\frac{2}{8}\right)>0
\end{aligned}
$$

что противоречит оптимальности $D$.

Лемма доказана.

Лемма 2. Если ИД D, соответствующее разбиению $\mathbf{R}=\left\{\mathbf{X}_{1}, \ldots, \mathbf{X}_{h+1}\right\}$ и решающее задачу $I \in \Phi(n, k)$, является оптимальным, то

$$
\left|\left\{i:\left|\mathbf{X}_{i}\right|=1, i=1, \ldots, h\right\}\right| \leqslant 1 \text {. }
$$

Доказательство. Допустим, что существуют по крайней мере два элемента разбиения $\mathbf{R}$, состоящих из одного элемента, $\mathbf{X}_{i}=\left\{x_{j}\right\}$ и $\mathbf{X}_{i+m}=\left\{x_{j^{\prime}}\right\}$. Рассмотрим разбиение $\mathbf{R}_{2}=\left\{\mathbf{X}_{1}, \ldots, \mathbf{X}_{i}^{\prime}, \ldots, \mathbf{X}_{h}^{\prime}\right\}$, множества $\mathbf{X}$, где $\mathbf{X}_{i}^{\prime}=\left\{x_{j}, x_{j^{\prime}}\right\}, \mathbf{X}_{p}^{\prime}=\mathbf{X}_{p}$ при 
$p=i+1, \ldots, i+m-1$ и $\mathbf{X}_{p}^{\prime}=\mathbf{X}_{p+1}$ при $p=i+m, \ldots, h$. Пусть $D_{2}-$ сбалансированное дерево, соответствующее разбиению $\mathbf{R}_{2}$ и решающее задачу $I$.

Заметим, что в сложностях деревьев $D$ и $D_{2}$ различаются только те группы слагаемых, которые соответствуют ярусам с номерами от $i$ до $i+m+1$ в дереве $D$ и ярусам с номерами от $i$ до $i+m$ в дереве $D_{2}$.

Рассмотрим группу слагаемых в $T(D)$, соответствующих ярусам с номерами $i, \ldots, i+m+1$. Так как $l_{i}=l_{i-1}+1, l_{j}=l_{i-1}+1+2(j-i), j=i+1, \ldots, i+m$, $l_{i+m+1}=l_{i-1}+2 m+2$, справедливы равенства

$$
\begin{aligned}
& 2^{l_{i}}\left(\frac{2}{3}\right)^{l_{i-1}}+\ldots+2^{l_{i+m+1}}\left(\frac{2}{3}\right)^{l_{i+m}} \\
& =2^{l_{i-1}}\left(\frac{2}{3}\right)^{l_{i-1}}\left(2+\sum_{p=1}^{m} 2^{2 p+1}\left(\frac{2}{3}\right)^{2 p-1}+2^{2 m+2}\left(\frac{2}{3}\right)^{2 m+1}\right) \\
& =\left(\frac{4}{3}\right)^{l_{i-1}}\left(2+2 \cdot \frac{3}{2} \sum_{p=1}^{m}\left(2 \cdot \frac{2}{3}\right)^{2 p}+2^{2} 2^{2 m} \frac{2}{3}\left(\frac{2}{3}\right)^{2 m}\right) \\
& =\left(\frac{4}{3}\right)^{l_{i-1}}\left(2+3 \sum_{p=1}^{m}\left(\frac{16}{9}\right)^{p}+\frac{8}{3}\left(\frac{16}{9}\right)^{m}\right) \\
& =\left(\frac{4}{3}\right)^{l_{i-1}}\left(2+3 \cdot \frac{16}{7}\left(\left(\frac{16}{9}\right)^{m+1}-1\right)+\frac{8}{3}\left(\frac{16}{9}\right)^{m}\right) \\
& =\left(\frac{4}{3}\right)^{l_{i-1}}\left(\frac{104}{7}\left(\frac{16}{9}\right)^{m}-\frac{34}{7}\right)^{m .}
\end{aligned}
$$

Рассмотрим теперь группу слагаемых в $T\left(D_{2}\right)$, соответствующих ярусам в $D_{2}$ с номерами $j \in\{i, \ldots, i+m\}$. Так как для любого номера $j$ на $j$-м ярусе находится $2^{l_{i-1}+2(j-i+1)}$ ребер, справедливы равенства

$$
\begin{aligned}
\sum_{p=0}^{m} 2^{l_{i-1}+2 p+2}\left(\frac{2}{3}\right)^{l_{i-1}+2 p} & =\left(\frac{4}{3}\right)^{l_{i-1}}\left(4 \sum_{p=0}^{m}\left(\frac{16}{9}\right)^{p}\right) \\
= & \left(\frac{4}{3}\right)^{l_{i-1}}\left(\frac{36}{7}\left(\left(\frac{16}{9}\right)^{m+1}-1\right)\right)=\left(\frac{4}{3}\right)^{l_{i-1}}\left(\frac{64}{7}\left(\frac{16}{7}\right)^{m}-\frac{36}{7}\right) .
\end{aligned}
$$

Тогда

$$
\begin{aligned}
T(D)-T\left(D_{2}\right) & =\left(\frac{4}{3}\right)^{l_{i-1}}\left(\left(\frac{16}{9}\right)^{m}\left(\frac{104}{7}-\frac{64}{7}\right)+\frac{2}{7}\right) \\
& =\left(\frac{4}{3}\right)^{l_{i-1}}\left(\frac{40}{7}\left(\frac{16}{9}\right)^{m}+\frac{2}{7}\right)>0 .
\end{aligned}
$$

Получаем, что исходное дерево не было оптимальным, что противоречит нашим предположениям. Следовательно, оптимальное дерево не может иметь более одного яруса, где из каждой вершины выходит по два ребра. Лемма доказана. 
Лемма 3. Если ИД D, соответствующее разбиению $\mathbf{R}=\left\{\mathbf{X}_{1}, \ldots, \mathbf{X}_{h+1}\right\}$ и решающее задачу $I \in \Phi(n, k)$, является оптимальным, то для всех $i=2, \ldots, h$ справедливо равенство $\left|\mathbf{X}_{i}\right|=2$.

Доказательство. Предположим, что в разбиении $\mathbf{R}$ есть два элемента $\mathbf{X}_{i}$ и $\mathbf{X}_{i+1}$, $i \in\{1, \ldots, h-1\}$, такие, что $\left|\mathbf{X}_{i}\right|=l_{i}-l_{i-1}=2$ и $\left|\mathbf{X}_{i+1}\right|=l_{i+1}-l_{i}=1$. Построим разбиение $\mathbf{R}_{3}$ множества $\mathbf{X}$ следующим образом: $\mathbf{R}_{3}=\left\{\mathbf{X}_{1}^{\prime}, \ldots, \mathbf{X}_{h+1}^{\prime}\right\}$, где $\mathbf{X}_{p}^{\prime}=\mathbf{X}_{p}$ при $p=1, \ldots, i-1$ и $p=i+2, \ldots, h+1$, а $\mathbf{X}_{i}^{\prime}=\mathbf{X}_{i+1}, \mathbf{X}_{i+1}^{\prime}=\mathbf{X}_{i}$. Рассмотрим сбалансированное дерево $D_{3}$, соответствующее разбиению $\mathbf{R}_{3}$.

Сложности $D$ и $D_{3}$ различаются только слагаемыми, соответствующими ярусам ребер с номерами $i$ и $i+1$. В $T(D)$ этим ярусам будут соответствовать слагаемые

$$
2^{l_{i-1}+2}\left(\frac{2}{3}\right)^{l_{i-1}}+2^{l_{i-1}+3}\left(\frac{2}{3}\right)^{l_{i-1}+2}=\left(\frac{4}{3}\right)^{l_{i-1}}\left(4+8 \cdot \frac{4}{9}\right)
$$

В $T\left(D_{3}\right)$ этим ярусам будут соответствовать слагаемые

$$
2^{l_{i-1}+1}\left(\frac{2}{3}\right)^{l_{i-1}}+2^{l_{i-1}+3}\left(\frac{2}{3}\right)^{l_{i-1}+1}=\left(\frac{4}{3}\right)^{l_{i-1}}\left(2+8 \cdot \frac{2}{3}\right)
$$

Следовательно,

$$
T(D)-T\left(D_{3}\right)=\left(\frac{4}{3}\right)^{l_{i-1}}\left(4+8 \cdot \frac{4}{9}-2-8 \cdot \frac{2}{3}\right)=\left(\frac{4}{3}\right)^{l_{i-1}} \frac{2}{9}>0 .
$$

Значит, дерево $D$ было не оптимальным, что противоречит нашим предположениям. Отсюда следует, что если в оптимальном дереве есть ярус, на котором из каждой вершины выходит по 2 ребра, то он может быть только первым.

Лемма доказана.

Лемма 4. Если ИД $D$, соответствующее разбиению $\mathbf{R}=\left\{\mathbf{X}_{1}, \ldots, \mathbf{X}_{h+1}\right\}$ и решающее задачу $I \in I(n, k)$, является оптимальным, то

$$
\sum_{j=1}^{h}\left|\mathbf{X}_{j}\right| \geqslant \log _{2} \frac{k}{6}
$$

Доказательство. Рассмотрим разбиение $\mathbf{R}_{4}=\left\{\mathbf{X}_{1}, \ldots, \mathbf{X}_{h},\left\{x_{j}\right\}, \mathbf{X}_{h+2}^{\prime}\right\},\left\{x_{j}\right\} \cap \mathbf{X}_{h+2}^{\prime}=\varnothing$, $\left\{x_{j}\right\} \cup \mathbf{X}_{h+2}^{\prime}=\mathbf{X}_{h+1}$.

Рассмотрим сбалансированное дерево $D_{4}$, соответствующее разбиению $\mathbf{R}_{4}$ и решающее задачу $I$.

В силу предположения оптимальности $D$, должно выполняться неравенство $T(D)-T\left(D_{4}\right) \leqslant 0$. Нетрудно видеть, что

$$
\begin{aligned}
T(D)-T\left(D_{4}\right) & =k\left(\frac{2}{3}\right)^{l_{h}}-2^{l_{h}+1}\left(\frac{2}{3}\right)^{l_{h}}-k\left(\frac{2}{3}\right)^{l_{h}+1} \\
& =k\left(\frac{2}{3}\right)^{l_{h}}\left(1-\frac{2^{l_{h}+1}}{k}-\frac{2}{3}\right)=k\left(\frac{2}{3}\right)^{l_{h}}\left(\frac{1}{3}-\frac{2^{l_{h}+1}}{k}\right)
\end{aligned}
$$


Отсюда следует, что должно выполняться неравенство

$$
l_{h}=\sum_{j=1}^{h}\left|\mathbf{X}_{j}\right| \geqslant \log _{2} \frac{k}{6}
$$

Лемма доказана.

Лемма 5. Если ИД $D$, соответствующее разбиению $\mathbf{R}=\left\{\mathbf{X}_{1}, \ldots, \mathbf{X}_{h+1}\right\}$ и решающее задачу $I \in \mathscr{I}(n, k)$, является оптимальным, то

$$
\sum_{j=1}^{h}\left|\mathbf{X}_{j}\right| \leqslant \log _{2} \frac{5 k}{9}
$$

Доказательство. Рассмотрим разбиение $\mathbf{R}_{6}=\left\{\mathbf{X}_{1}, \ldots, \mathbf{X}_{h-1}, \mathbf{X}_{h}^{\prime}\right\}$, где $\mathbf{X}_{h}^{\prime}=\mathbf{X}_{h} \cup \mathbf{X}_{h+1}$. Пусть дерево $D_{6}$ соответствует разбиению $\mathbf{R}_{6}$ и решает задачу $I$, тогда

$$
\begin{aligned}
T(D)-T\left(D_{6}\right) & =2^{l_{h}}\left(\frac{2}{3}\right)^{l_{h-1}}+k\left(\frac{2}{3}\right)^{l_{h}}-k\left(\frac{2}{3}\right)^{l_{h-1}} \\
& =k\left(\frac{2}{3}\right)^{l_{h-1}}\left(\frac{2^{l_{h}}}{k}+\left(\frac{2}{3}\right)^{l_{h}-l_{h-1}}-1\right) .
\end{aligned}
$$

Учтем, что $l_{h}-l_{h-1}=2$ при $k>12$ (это следует из того, что для оптимального дерева должно выполняться неравенство $l_{h} \geqslant \log _{2}(k / 6)$ и при $k>12$ выполнено неравенство $l_{h} \geqslant 2$ ), тогда

$$
T(D)-T\left(D_{6}\right)=k\left(\frac{2}{3}\right)^{l_{h-1}}\left(\frac{2^{l_{h}}}{k}-\frac{5}{9}\right) .
$$

Следовательно, $l_{h} \leqslant \log _{2}(5 k / 9)$.

Непосредственной проверкой легко удостовериться, что при $1 \leqslant k \leqslant 6$ оптимальное дерево задается разбиением $\mathbf{R}=\{\mathbf{X}\}$ (то есть $l_{h}=0$ ), при $7 \leqslant k \leqslant 11$ оптимальное дерево задается разбиением $\mathbf{R}=\left\{\mathbf{X}_{1}, \mathbf{X}_{2}\right\}$, где $\left|\mathbf{X}_{1}\right|=1$ (то есть $l_{h}=1$ ), и при $k=12$ оптимальное дерево задается разбиением $\mathbf{R}=\left\{\mathbf{X}_{1}, \mathbf{X}_{2}\right\}$, где $\left|\mathbf{X}_{1}\right|=2$ (то есть $l_{h}=2$ ).

Лемма доказана.

Подводя итоги рассуждений, приведем алгоритм построения оптимального дерева.

Деревом $D_{l}$ с оптимальной $l$-структурой назовем ИД, соответствующее разбиению $\mathbf{R}_{l}=\left\{\mathbf{X}_{1}, \ldots, \mathbf{X}_{m}, \mathbf{X}_{m+1}\right\}$, где $\left.m=\right] l / 2\left[,\left|\mathbf{X}_{i}\right|=2\right.$ при $i=2, \ldots, m,\left|\mathbf{X}_{1}\right|=1$, если $l$ нечетно, и $\left|\mathbf{X}_{1}\right|=2$, если $l$ четно. Вычислим сложность дерева с оптимальной $l$-структурой.

Если $l=2 m$, то

$$
\begin{aligned}
T\left(D_{2 m}\right) & =\sum_{i=1}^{m} 2^{2 i}\left(\frac{2}{3}\right)^{2 i-2}+k\left(\frac{2}{3}\right)^{2 m} \\
& =\frac{9}{4} \sum_{i=1}^{m}\left(\frac{16}{9}\right)^{i}+k\left(\frac{2}{3}\right)^{2 m}=\frac{36}{7}\left(\frac{4}{3}\right)^{2 m}+k\left(\frac{2}{3}\right)^{2 m}-\frac{36}{7}
\end{aligned}
$$


Если $l=2 m+1$, то

$$
\begin{aligned}
T\left(D_{2 m+1}\right) & =2+\sum_{i=1}^{m} 2^{2 i+1}\left(\frac{2}{3}\right)^{2 i-1}+k\left(\frac{2}{3}\right)^{2 m+1}=2+3 \sum_{i=1}^{m}\left(\frac{16}{9}\right)^{i}+k\left(\frac{2}{3}\right)^{2 m+1} \\
& =2+\frac{48}{7}\left(\frac{16}{9}\right)^{m}-\frac{48}{7}+k\left(\frac{2}{3}\right)^{2 m+1}=\frac{48}{7}\left(\frac{16}{9}\right)^{m}+k\left(\frac{2}{3}\right)^{2 m+1}-\frac{34}{7} \\
& =\frac{36}{7}\left(\frac{4}{3}\right)^{2 m+1}+k\left(\frac{2}{3}\right)^{2 m+1}-\frac{34}{7}
\end{aligned}
$$

Таким образом,

$$
T\left(D_{l}\right)=\frac{36}{7}\left(\frac{4}{3}\right)^{l}+k\left(\frac{2}{3}\right)^{l}-d
$$

где $d=34 / 7$, если $l$ нечетно, $d=36 / 7$, если $l$ четно.

Рассматриваем целые числа на отрезке $\left[\log _{2}(k / 6), \log _{2}(5 k / 9)\right]$. В этих пределах всегда есть одно или два целых числа, так как

$$
\log _{2} \frac{5 k}{9}-\log _{2} \frac{k}{6}=\log _{2} \frac{10}{3} \approx 1,7 .
$$

Если в этот промежуток попало только одно целое четное число $2 m$, то оптимальным является решающее задачу ИД $D_{2 m}$, высота которого равна $m+1$ и из каждой вершины всех ярусов, кроме последнего выходит по 4 ребра.

Если в этот промежуток попало только одно целое нечетное число $2 m+1$, то оптимальным является решающее задачу ИД $D_{2 m+1}$, высота которого $m+2$, на первом ярусе 2 ребра, на последующих $m$ ярусах по 4 ребра из вершины, и на последнем суммарно $k$ ребер.

Если в отрезок попали два целых последовательных числа $l$ и $l+1$ и $T\left(D_{l}\right) \leqslant T\left(D_{l+1}\right)$, то оптимальным деревом является $D_{l}$, в противном случае $D_{l+1}$.

Пусть в отрезок $\left[\log _{2}(k / 6), \log _{2}(5 k / 9]\right.$ попали два целых числа $l$ и $l+1$. Тогда, очевидно, что

$$
\log _{2} \frac{k}{6} \leqslant l \leqslant \log _{2} \frac{5 k}{18}, \quad \log _{2} \frac{k}{3} \leqslant l+1 \leqslant \log _{2} \frac{5 k}{9} .
$$

Если в отрезке $\left[\log _{2}(k / 6), \log _{2}(5 k / 9]\right.$ находится только одно целое число, то оно принадлежит интервалу $\left(\log _{2}(5 k / 18), \log _{2}(k / 3)\right)$.

Пусть $l=2 m$. Рассмотрим разность

$$
T\left(D_{2 m+1}\right)-T\left(D_{2 m}\right)=\frac{2}{7}+\frac{12}{7}\left(\frac{4}{3}\right)^{2 m}-\frac{1}{3} k\left(\frac{2}{3}\right)^{2 m}=\frac{2}{7}+\left(\frac{2}{3}\right)^{2 m}\left(\frac{12}{7} 2^{2 m}-\frac{k}{3}\right) .
$$

Так как $2 m \in\left[\log _{2}(k / 6), \log _{2}(5 k / 18)\right]$, можно положить $2 m=\log _{2} a k$, где параметр $a \in[(1 / 6,5 / 18)]$. Тогда $2^{2 m}=a k,(2 / 3)^{2 m}=(a k)^{\log _{2}(2 / 3)}$ и

$$
\begin{aligned}
T\left(D_{2 m+1}\right)-T\left(D_{2 m}\right) & =\frac{2}{7}+(a k)^{\log _{2}(2 / 3)}\left(\frac{12}{7} a k-\frac{k}{3}\right) \\
& =\frac{2}{7}+a^{\log _{2}(2 / 3)} k^{\log _{2}(4 / 3)}\left(\frac{12}{7} a-\frac{1}{3}\right) .
\end{aligned}
$$


Очевидно, что если $a \geqslant 7 / 36$, то $T\left(D_{2 m+1}\right)-T\left(D_{2 m}\right)>0$, то есть, если $\log _{2}(7 k / 36) \leqslant 2 m \leqslant \log _{2}(5 k / 18)$, то $D_{2 m}$ является оптимальным.

Для того, чтобы дерево $D_{2 m}$ было оптимальным и при $a<7 / 36$, необходимо, чтобы выполнялось неравенство

$$
\frac{2}{7}+a^{\log _{2}(2 / 3)} k^{\log _{2}(4 / 3)}\left(\frac{12}{7} a-\frac{1}{3}\right) \geqslant 0,
$$

то есть

$$
k \leqslant\left(\frac{2 / 7}{(a)^{\log _{2}(2 / 3)}(1 / 3-12 a / 7)}\right)^{\log _{4 / 3} 2} .
$$

Очевидно, что минимум по $a$ на полуинтервале $[1 / 6,7 / 36)$ выражение в правой части неравенства принимает при $a=1 / 6$, и он равен

$$
\left(\frac{2 / 7}{\left.(1 / 6)^{\log _{2}(2 / 3)}\right) / 21}\right)^{\log _{4 / 3} 2}=6,
$$

при $a \rightarrow 7 / 36$ выражение в правой части неравенства стремится к бесконечности. Поэтому при $2 m \in\left[\log _{2}(k / 6), \log _{2}(7 k / 36)\right)$ знак выражения $T\left(D_{2 m+1}\right)-T\left(D_{2 m}\right)$ зависит or $k$.

Пусть $l=2 m-1$. Рассмотрим разность

$$
\begin{aligned}
T\left(D_{2 m-1}\right)-T\left(D_{2 m}\right) & =\frac{2}{7}+\frac{k}{3}\left(\frac{2}{3}\right)^{2 m-1}-\frac{12}{7}\left(\frac{4}{3}\right)^{2 m-1} \\
& =\frac{2}{7}+\left(\frac{2}{3}\right)^{2 m-1}\left(\frac{k}{3}-\frac{12}{7} 2^{2 m-1}\right)
\end{aligned}
$$

Положим $2 m-1=\log _{2} a k$, тогда

$$
T\left(D_{2 m-1}\right)-T\left(D_{2 m}\right)=\frac{2}{7}+(a k)^{\log _{2}(2 / 3)} k^{\log _{2}(4 / 3)}\left(\frac{1}{3}-\frac{12}{7} a\right) .
$$

Очевидно, что при $a \in[1 / 6,7 / 36)$ это выражение положительно, и поэтому дерево $D_{2 m}$ является оптимальным.

Для неотрицательности $T\left(D_{2 m-1}\right)-T\left(D_{2 m}\right)$ при $a \in[7 / 36,5 / 18]$ необходимо и достаточно, чтобы выполнялось неравенство

$$
k \leqslant\left(\frac{2 / 7}{(a)^{\log _{2}(2 / 3)}(12 a / 7-1 / 3)}\right)^{\log _{4 / 3} 2} .
$$

Минимум в правой части неравенства достигается при $a=5 / 18$, и он равен

$$
\left(2\left(\frac{18}{5}\right)^{\log _{2}(2 / 3)}\right)^{\log _{4 / 3} 2} \approx 1
$$

при стремлении $a$ к 7/36 выражение в правой части неравенства стремится к бесконечности. Следовательно, знак разности $T\left(D_{2 m-1}\right)-T\left(D_{2 m}\right)$ при $2 m-1$, удовлетворяющем неравенствам $\log _{2}(7 k / 36) \leqslant 2 m-1 \leqslant \log _{2}(5 k / 18)$, зависит от $k$.

Таким образом, для того чтобы определить параметр $l_{h}$ оптимального дерева, достаточно проверить несколько простых неравенств, а не вычислять два выражения для сложностей (требующих возведения $k$ в степень), что может быть удобным при больших $k$. 


\section{4. Доказательство теоремы 1}

Выясним порядок найденной сложности. Рассмотрим наименьшее целое число $l$ из отрезка $\left[\log _{2}(k / 6), \log _{2}(5 k / 9)\right]$, очевидно, что оно попадет в полуинтервал $\left[\log _{2}(k / 6), \log _{2}(k / 3)\right)$. Пусть $a=2^{l} / k, d_{1}$ и $d_{2}$ - различные числа из множества $\{-34 / 7,-36 / 7\}$, тогда $l=\log _{2} a k$, $a \in[1 / 6,1 / 3)$. Сложность дерева с оптимальной $l$-структурой согласно (3) равна

$$
\begin{aligned}
T\left(D_{l}\right) & =\frac{36}{7}\left(\frac{4}{3}\right)^{l}+k\left(\frac{2}{3}\right)^{l}+d_{1}=\frac{36}{7}\left(\frac{4}{3}\right)^{\log _{2} a k}+k\left(\frac{2}{3}\right)^{\log _{2} a k}+d_{1} \\
& =\frac{36}{7} a^{\log _{2}(4 / 3)} k^{\log _{2}(4 / 3)}+a^{\log _{2}(2 / 3)} k^{\log _{2}(4 / 3)}+d_{1} \\
& =k^{\log _{2}(4 / 3)}\left(\frac{36}{7} a^{\log _{2}(4 / 3)}+a^{\log _{2}(2 / 3)}\right)+d_{1}
\end{aligned}
$$

Сложность дерева с оптимальной $(l+1)$-структурой равна

$$
\begin{aligned}
T\left(D_{l+1}\right) & =\frac{36}{7}\left(\frac{4}{3}\right)^{l+1}+k\left(\frac{2}{3}\right)^{l+1}+d_{2}=\frac{36}{7} \frac{4}{3}\left(\frac{4}{3}\right)^{\log _{2} a k}+k \frac{2}{3}\left(\frac{2}{3}\right)^{\log _{2} a k}+d_{2} \\
& =\frac{48}{7} a^{\log _{2}(4 / 3)} k^{\log _{2}(4 / 3)}+\frac{2}{3} a^{\log _{2}(2 / 3)} k^{\log _{2}(4 / 3)}+d_{2} \\
& =k^{\log _{2}(4 / 3)}\left(\frac{48}{7} a^{\log _{2}(4 / 3)}+\frac{2}{3} a^{\log _{2}(2 / 3)}\right)+d_{2} .
\end{aligned}
$$

Таким образом, если для задачи $I$ оптимальным является дерево $D_{l}$, то

$$
T_{d}(I)=k^{\log _{2}(4 / 3)}\left(\frac{36}{7} a^{\log _{2}(4 / 3)}+a^{\log _{2}(2 / 3)}\right)+d_{1},
$$

а если оптимально дерево $D_{l+1}$, то

$$
T_{d}(I)=k^{\log _{2}(4 / 3)}\left(\frac{48}{7} a^{\log _{2}(4 / 3)}+\frac{2}{3} a^{\log _{2}(2 / 3)}\right)+d_{2} .
$$

Рассмотрим разность

$$
\begin{aligned}
k^{\log _{2}(4 / 3)}\left(\frac{36}{7} a^{\log _{2}(4 / 3)}+a^{\log _{2}(2 / 3)}\right)+d_{1}- & \left(k^{\log _{2}(4 / 3)}\left(\frac{48}{7} a^{\log _{2}(4 / 3)}+\frac{2}{3} a^{\log _{2}(2 / 3)}\right)+d_{2}\right) \\
& =k^{2-\log _{2} 3}\left(\frac{12}{7} a^{2-\log _{2} 3}-\frac{1}{3} a^{1-\log _{2} 3}\right)+d
\end{aligned}
$$

При $a>7 / 36$ это выражение положительно, при $a<7 / 36$ отрицательно. Следовательно,

$$
k^{2-\log _{2} 3} \inf _{a \in[1 / 6,1 / 3)} A(a)-\frac{36}{7} \leqslant T_{d}(I) \leqslant k^{2-\log _{2} 3} \sup _{a \in[1 / 6,1 / 3)} A(a)-\frac{34}{7},
$$

где

$$
A(a)= \begin{cases}(48 / 7) a^{\log _{2}(4 / 3)}+(2 / 3) a^{\log _{2}(2 / 3)}, & \text { если } a \in[1 / 6,7 / 36), \\ (36 / 7) a^{\log _{2}(4 / 3)}+a^{\log _{2}(2 / 3)}, & \text { если } a \in[7 / 36,1 / 3) .\end{cases}
$$


Найдем наибольшее и наименьшее значения функщии $A(a)$ на отрезке $[1 / 6,1 / 3]$. Введем обозначения

$$
\begin{aligned}
& A_{1}(a)=\frac{48}{7} a^{\log _{2}(4 / 3)}+\frac{2}{3} a^{\log _{2}(2 / 3)} \\
& A_{2}(a)=\frac{36}{7} a^{\log _{2}(4 / 3)}+a^{\log _{2}(2 / 3)}
\end{aligned}
$$

Тогда

$$
\begin{aligned}
\min _{a \in[1 / 6,1 / 3]} A(a) & =\min \left(\min _{a \in[1 / 6,7 / 36]} A_{1}(a), \min _{a \in[7 / 36,1 / 3]} A_{2}(a)\right), \\
\max _{a \in[1 / 6,1 / 3]} A(a) & =\max \left(\max _{a \in[1 / 6,7 / 36]} A_{1}(a), \max _{a \in[7 / 36,1 / 3]} A_{2}(a)\right) .
\end{aligned}
$$

Исследуем функцию $A_{1}(a)$ на отрезке [1/6,7/36]. Нетрудно видеть, что

$$
A_{1}^{\prime}(\hat{a})=0 \Longleftrightarrow \frac{48}{7} \hat{a}^{\log _{2}(4 / 3)}+\frac{2}{3} \hat{a}^{\log _{2}(2 / 3)}=0 \Longleftrightarrow \hat{a}=\frac{7}{72} \log _{4 / 3} \frac{3}{2} .
$$

Следовательно, $\hat{a}<1 / 6$ и $A_{1}^{\prime}(a)>0$ при $a>\hat{a}$. Значит, $A_{1}(a)$ возрастает при $a \in[1 / 6,7 / 36]$,

$$
\begin{aligned}
& \min _{a \in[1 / 6,7 / 36]} A_{1}(a)=A_{1}(1 / 6)=\frac{38}{21}(1 / 6)^{\log _{2}(2 / 3)}=\frac{19}{7}(1 / 3)^{\log _{2}(2 / 3)} \approx 5,16, \\
& \max _{a \in[1 / 6,7 / 36]} A_{1}(a)=A_{1}(7 / 36)=2(7 / 36)^{\log _{2}(2 / 3)} \approx 5,2 .
\end{aligned}
$$

Исследуем функцию $A_{2}(a)$ на отрезке [7/36, 1/3]. Нетрудно видеть, что

$$
A_{2}^{\prime}(\hat{a})=0 \Longleftrightarrow \frac{36}{7} \hat{a}^{\log _{2}(4 / 3)}+\hat{a}^{\log _{2}(2 / 3)}=0 \Longleftrightarrow \hat{a}=\frac{7}{36} \log _{4 / 3}(3 / 2),
$$

причем $A_{2}(a)<0$ при $a<\hat{a}, A_{2}(a)>0$ при $a>\hat{a}$ и $7 / 36<\hat{a}<1 / 3$. Значит,

$$
\begin{aligned}
\min _{[7 / 36,1 / 3]} A_{2}(a) & =A_{2}(\hat{a})=\log _{4 / 3} 2\left(\frac{7}{36} \log _{4 / 3} \frac{3}{2}\right)^{\log _{2}(2 / 3)} \approx 5,13, \\
\max _{[7 / 36,1 / 3]} A_{2}(a) & =\max \left(A_{2}(7 / 36), A_{2}(1 / 3)\right),
\end{aligned}
$$

причем $A_{2}(7 / 36)=A_{1}(7 / 36)$, а $A_{2}(1 / 3)=(19 / 7)(1 / 3)^{\log _{2}(2 / 3)}=A_{1}(1 / 6)$, то есть

$$
\left.\max _{[7 / 36,1 / 3]} A_{2}(a)=A_{2}(7 / 36)\right)=2(7 / 36)^{1-\log _{2} 3} \text {. }
$$

Итак, наибольшее значение $A(a)$ на $[1 / 6,1 / 3)$ достигается при $a=7 / 36$ и

$$
\max _{a \in[1 / 6,1 / 3)} A(a)=2(7 / 36)^{1-\log _{2} 3} .
$$

Наименьшее значение $A(a)$ на $[1 / 6,1 / 3)$ достигается при $a=(7 / 36) \log _{4 / 3}(3 / 2)$ и

$$
\min _{a \in[1 / 6,1 / 3)} A(a)=\log _{4 / 3} 2\left((7 / 36) \log _{4 / 3}(3 / 2)^{1-\log _{2} 3} .\right.
$$

Значит,

$$
k^{2-\log _{2} 3} \log _{4 / 3} 2\left(\frac{7}{36} \log _{4 / 3} \frac{3}{2}\right)^{1-\log _{2} 3}-\frac{36}{7} \leqslant T_{d}(I) \leqslant k^{2-\log _{2} 3} 2\left(\frac{7}{36}\right)^{1-\log _{2} 3}-\frac{34}{7} .
$$

Тем самым теорема 1 доказана. 


\section{5. Доказательство теоремы 2}

Для каждого числа вида $a=2^{q} / p$ найдется последовательность натуральных чисел $k_{i}$, $i=0,1,2, \ldots$, таких, что $l_{i}=\log _{2} a k_{i}$ является целым числом, а именно, $k_{i}=2^{i} p$, $l_{i}=q+i$.

Лемма 6. Для любого $\varepsilon>0$ и любого $n / m \in[1 / 6,1 / 3]$, где $n$ и $m$ - натуральные числа, существуют натуральные $q$ и р такие, что

$$
\left|\frac{2^{q}}{p}-\frac{n}{m}\right|<\varepsilon .
$$

Доказательство. Для каждого $q=1,2, \ldots$ рассмотрим последовательности вида $2^{q} /\left(3 \cdot 2^{q}+r\right), r=0,1,2, \ldots$ Для того, чтобы выполнялось включение $2^{q} /\left(3 \cdot 2^{q}+r\right) \epsilon$ $[1 / 6,1 / 3]$, необходимо, чтобы выполнялись неравенства $0 \leqslant r \leqslant 3 \cdot 2^{q}$. Понятно, что для каждого $q$ последовательность является убывающей по $r$, а также, что с ростом $r$ убывает расстояние между соседними членами последовательности, то есть, максимальное расстояние $\Delta_{q}$ будет между членами $2^{q} / 3 \cdot 2^{q}+0=1 / 3$ и $2^{q} / 3 \cdot 2^{q}+1$, и оно равно

$$
\Delta_{q}=\frac{1}{3}-\frac{2^{q}}{3 \cdot 2^{q}+1}=\frac{1}{9 \cdot 2^{q}+3} .
$$

Найдем $q$, при которых $\Delta_{q}<\varepsilon$, то есть $9 \cdot 2^{q}>1 / \varepsilon, q>\log _{2}(1 / 9 \varepsilon)$. Теперь для $q=] \log _{2}(1 / 9 \varepsilon)$ [ выберем такое $r$, что выполнены неравенства

$$
\begin{aligned}
\frac{2^{q}}{3 \cdot 2^{q}+r+1} & \leqslant \frac{n}{m} \leqslant \frac{2^{q}}{3 \cdot 2^{q}+r}, \\
3 \cdot 2^{q}+r & \leqslant \frac{m}{n} 2^{q} \leqslant 3 \cdot 2^{q}+r+1, \\
r & \leqslant 2^{q}\left(\frac{m}{n}-3\right) \leqslant r+1 .
\end{aligned}
$$

Отсюда следует, что $r=\left[2^{q}(m / n-3)\right]$.

Лемма доказана.

Множество рациональных чисел всюду плотно в $\mathbf{R}$, поэтому и для любого $\varepsilon>0$, любого действительного числа $b$ из некоторого отрезка существует число вида $2^{q} / p$ такое, что $\left|b-2^{q} / p\right|<\varepsilon$. Функщия $A(a)$ непрерывна на $[1 / 6,1 / 3)$, поэтому для любого $\varepsilon>0$ существует $\delta$ такое, что $|A(x)-A(y)|<\varepsilon$ при $|x-y|<\delta$. Если положить

$$
\begin{aligned}
& c_{1}=\log _{4 / 3} 2\left(\frac{7}{36} \log _{4 / 3} \frac{3}{2}\right)^{1-\log _{2} 3}, \\
& c_{2}=2\left(\frac{7}{36}\right)^{1-\log _{2} 3},
\end{aligned}
$$

то получим, что для любого числа из $\left[c_{1}, c_{2}\right]$ и любого положительного $\varepsilon$ найдется число $b \in(a-\varepsilon, a+\varepsilon), b=A\left(2^{q} / p\right)$, такое, что существует возрастающая последовательность натуральных чисел $k_{i}=2^{i} p$, для которой $T_{d}\left(I_{i}\right) \sim b k_{i}^{2-\log _{2} 3}$ при $i \rightarrow \infty$ и $I_{i} \in I\left(n, k_{i}\right)$.

Теорема доказана. 


\section{Список литературы}

1. Блайвас Т. Д., Решение задачи интервального поиска на булевом кубе. В сб.: Тезисы докладов ХІІІ Междуиародной коиферепчии «Проблемы теоретической кибериетики». Казань, 2002, с. 22.

2. Гасанов Э. Э., Кудрявцев В. Б., Теория храпения и поиска ииформачии. Физматлит, Москва, 2002.

Статья поступила 24.06.2003. 